\title{
On Measuring the Quality of a Network Community Structure
}

\author{
Mingming Chen \\ Department of Computer Science \\ Rensselaer Polytechnic Institute \\ 110 8th Street, Troy, NY 12180 \\ Email: chenm8@rpi.edu
}

\author{
Tommy Nguyen \\ Department of Computer Science \\ Rensselaer Polytechnic Institute \\ 110 8th Street, Troy, NY 12180 \\ Email: nguyet11@rpi.edu
}

\author{
Boleslaw K. Szymanski \\ Department of Computer Science \\ Rensselaer Polytechnic Institute \\ 110 8th Street, Troy, NY 12180 \\ Email: szymab@rpi.edu
}

\begin{abstract}
Modularity is widely used to effectively measure the strength of the community structure found by community detection algorithms. However, modularity maximization suffers from two opposite yet coexisting problems: in some cases, it tends to favor small communities over large ones while in others, large communities over small ones. The latter tendency is known in the literature as the resolution limit problem. To address them, we propose to modify modularity by subtracting from it the fraction of edges connecting nodes of different communities and by including community density into modularity. We refer to the modified metric as Modularity Density and we demonstrate that it indeed resolves both problems mentioned above. We describe the motivation for introducing this metric by using intuitively clear and simple examples. We also discuss the results of applying this metric, modularity, and several other popular community quality metrics to two real dynamic networks. The results imply that Modularity Density is consistent with all the community quality measurements but not modularity, which suggests that Modularity Density is an improved measurement of the community quality compared to modularity.
\end{abstract}

\section{INTRODUCTION}

Communities are basic structures in sociology intensively studied since 1950's [1], [2]. Social media enabled online communities that link people regardless of their physical location. Thus, computational methods are needed to analyze and detect such large communities. Formally, communities are defined as groups of nodes within which connections are denser than between them [3] and community detection became one of the fundamental issues in network science. Community detection has been shown to reveal latent yet meaningful structure not only for groups in online and contact-based social networks, but also in functional modules in protein-protein interaction networks, groups of customers with similar interests in online retailer user networks, groups of scientists in interdisciplinary collaboration networks, etc. [4].

In the last decade, the most popular community detection method, proposed by Newman [5], has been to maximize the quality metric known as modularity [3], [6] over all the possible partitions of a network. This metric measures the difference (relative to the total number of edges) between the actual and expected (in a randomized graph with the same number of nodes and the same degree distribution) number of edges within a given community. It is widely used to measure the strength of the community structures detected by the community detection algorithms. However, modularity maximization has two opposite yet concurrent problems. In some cases, it tends to split large communities into smaller communities. In other cases, it tends to form large communities by merging communities that are smaller than a certain threshold which depends on the total number of edges in the network and on the degree of inter-connectivity between the communities. The latter problem is known as the resolution limit problem [7].

To solve these two problems simultaneously, we propose a new community quality metric, that we termed Modularity Density, as an alternative to modularity. First, we show that modularity decreased by Split Penalty, defined as the fraction of edges that connect nodes of different communities, solves the problem of favoring small communities. Next, we demonstrate that including community density into modularity addresses the problem of favoring large communities. We refer to the resulting metric as Modularity Density.

We discuss our experiments with Modularity Density, modularity, and other popular community quality metrics, including the number of Intra-edges, Contraction, the number of Inter-edges, Expansion, and Conductance [8], on two real dynamic networks. The results show that Modularity Density is different from original modularity, but consistent with all those community quality measurements, which implies that Modularity Density is effective in measuring the community quality of networks.

The rest of the paper is organized as follows. First, in Section II we discuss some related works. Then, we briefly introduce modularity and illustrate our motivation to propose the new metric with examples in Section III. Section IV presents the experiments that demonstrate Modularity Density solves the two problems of modularity simultaneously. Finally, we conclude and discuss the future work in Section V.

\section{RELATED WORK}

Several metrics for evaluating the quality of community structure have been introduced. The most popular and widely used is modularity [3], [6]. It is defined as the difference (relative to the total number of edges) between the actual and expected (in a randomized graph with the same number of nodes and the same degree sequence) number of edges inside a given community. Although initially defined for unweighted and undirected networks, the definition of modularity has been subsequently extended to capture community structure in weighted networks [9] and then in directed networks [10].

However, recently, Fortunato and Barthélemy [7] presented a resolution limit problem of modularity, essence of which is that optimizing modularity will not find communities smaller than a threshold size, or weight [11]. This threshold depends on the total number, or total weight, of edges in the network and on the degree of interconnectedness between the communities. Moreover, Good et al. [12] shown that the range of modularity values computed over all possible partitions of a graph has a 


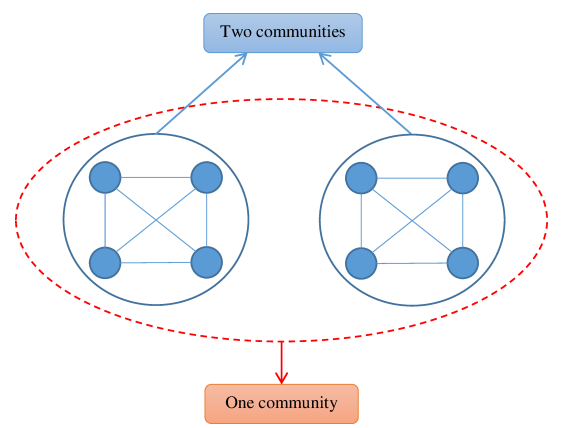

(a) Two very well separated communities.

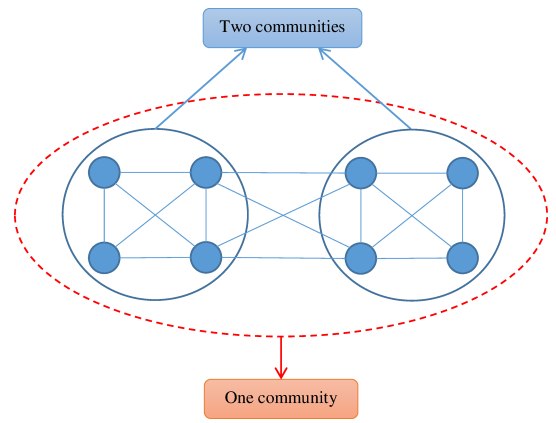

(d) Ambiguity between one and two communities.

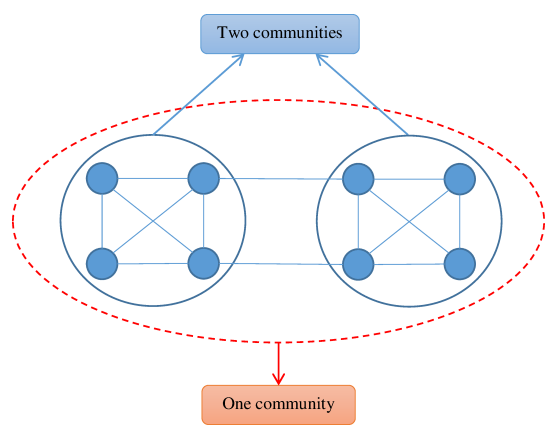

(b) Two well separated communities.

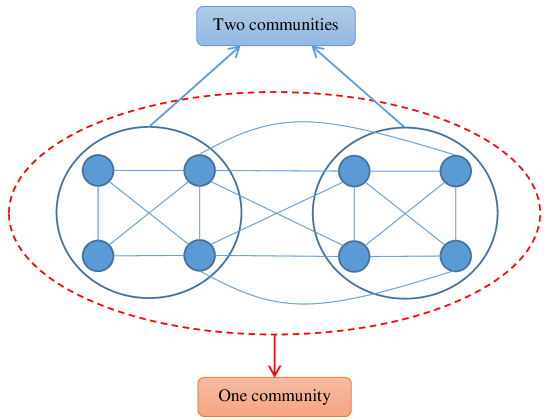

(e) One well connected community.

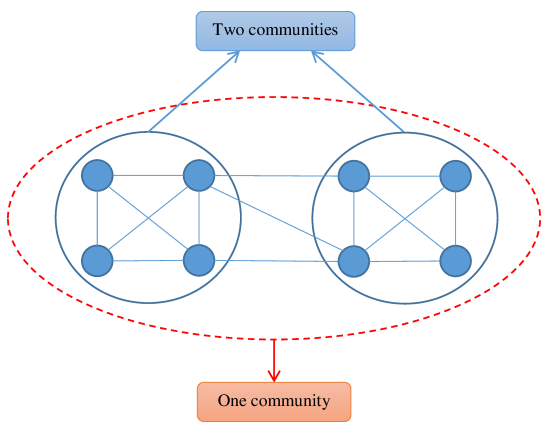

(c) Two weakly connected communities.

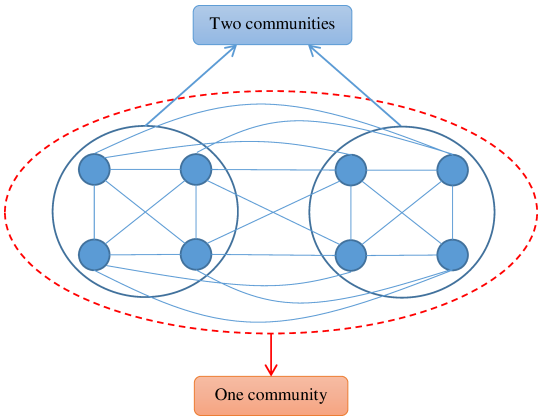

(f) One very well connected community.

Fig. 1. Six simple network examples that have two different community structures, one with a single big community containing all eight nodes and the other with the two small communities each containing four different nodes.

structure in which the maximum modularity partition is typically concealed among an exponentially large (in terms of the graph size) number of structurally dissimilar, high-modularity partitions. To address this resolution limit problem, multiresolution versions of modularity [13], [14] were proposed to allow researchers to specify a tunable target resolution limit parameter and identify communities on that scale. Typically, it is not clear how to choose the correct value for this parameter. Furthermore, Lancichinetti and Fortunato [15] stated that even those multi-resolution versions of modularity as well as its original version are not only inclined to merge the smallest wellformed communities but also to split the largest well-formed communities. In contrast, the Modularity Density metric we propose here solves those two problems of modularity without the trouble of specifying any particular parameter.

\section{Modularity Density}

In this section, we first formally introduce Newman's definition of modularity and then illustrate the motivation for modifying modularity with several simple network examples. Next, we propose a new community quality metric, called Modularity Density, as an alternative to modularity by combining modularity with Split Penalty and community density to avoid the two coexisting problems of modularity. Finally, we define Modularity Density for different kinds of networks, including unweighted and undirected networks, weighted networks, and directed networks, based on the corresponding formulas of modularity.

\section{A. Newman's Modularity}

Modularity [3], [6] for unweighted and undirected networks is defined as the ratio of difference between the actual and expected (in a randomized graph with the same number of nodes and the same degree sequence) number of edges within the community. For the given community partition of a network $G=(V, E)$ with $|E|$ edges, modularity $(Q)$ [3] is given by

$$
Q=\sum_{c_{i} \in C}\left[\frac{\left|E_{c_{i}}^{\text {in }}\right|}{|E|}-\left(\frac{2\left|E_{c_{i}}^{\text {in }}\right|+\left|E_{c_{i}}^{\text {out }}\right|}{2|E|}\right)^{2}\right],
$$

where $C$ is the set of all the communities, $c_{i}$ is a specific community in $C,\left|E_{c_{i}}^{i n}\right|$ is the number of edges between nodes within community $c_{i}$, and $\left|E_{c_{i}}^{o u t}\right|$ is the number of edges from the nodes in community $c_{i}$ to the nodes outside $c_{i}$.

The definition of modularity [9] for the weighted networks has precisely the same formula, Equation (1), as for the unweighted and undirected networks. However, for weighted networks, $|E|$ is the sum of the weights of all the edges in the network, $\left|E_{c_{i}}^{i n}\right|$ is the sum of the weights of the edges between nodes within community $c_{i}$, and $\left|E_{c_{i}}^{\text {out }}\right|$ is the sum of the weights of the edges from the nodes in community $c_{i}$ to the nodes outside $c_{i}$.

The formula of modularity for directed networks [10] is as follows

$$
Q=\sum_{c_{i} \in C}\left[\frac{\left|E_{c_{i}}^{i n}\right|}{|E|}-\frac{\left(\left|E_{c_{i}}^{i n}\right|+\left|E_{\text {out }, c_{i}}\right|\right)\left(\left|E_{c_{i}}^{i n}\right|+\left|E_{c_{i}, \text { out }}\right|\right)}{|E|^{2}}\right],
$$

where $\left|E_{\text {out }, c_{i}}\right|$ is the number of edges from the nodes outside community $c_{i}$ to the nodes in community $c_{i}$ and $\left|E_{c_{i}, \text { out }}\right|$ is the number of edges from the nodes in community $c_{i}$ to the nodes outside $c_{i}$. For undirected networks, it is clear that $\left|E_{\text {out }, c_{i}}\right|=$ $\left|E_{c_{i}, \text { out }}\right|=\left|E_{c_{i}}^{\text {out }}\right|$ and thus the directed modularity is reduced to undirected modularity.

\section{B. Motivation for Introducing Split Penalty}

In this subsection, we demonstrate the motivation for introducing Split Penalty into modularity by using seven intuitively clear and simple network examples, six of which are presented 
TABLE I. METRIC VAlues OF THE EXAMPLE: Two Very Well SEPARATED COMMUNITIES

\begin{tabular}{l||c|c|c|c}
\hline \hline & Modularity $(Q)$ & Split Penalty $(S P)$ & $Q_{s}$ & $Q_{d s}$ \\
\hline Two communities & 0.5 & 0 & 0.5 & 0.5 \\
\hline One community & 0 & 0 & 0 & 0.245 \\
\hline \hline
\end{tabular}

TABLE II. METRIC VALUES OF THE EXAMPLE: TWO WELL SEPARATED COMMUNITIES.

\begin{tabular}{l||c|c|c|c}
\hline \hline & Modularity $(Q)$ & Split Penalty $(S P)$ & $Q_{s}$ & $Q_{d s}$ \\
\hline Two communities & 0.357 & 0.143 & 0.214 & 0.339 \\
\hline One community & 0 & 0 & 0 & 0.25 \\
\hline \hline
\end{tabular}

TABLE III. METRIC VALUES OF THE EXAMPLE: TwO WEAKLY CONNECTED COMMUNITIES.

\begin{tabular}{l||c|c|c|c}
\hline \hline & Modularity $(Q)$ & Split Penalty $(S P)$ & $Q_{s}$ & $Q_{d s}$ \\
\hline Two communities & 0.3 & 0.2 & 0.1 & 0.263 \\
\hline One community & 0 & 0 & 0 & 0.249 \\
\hline \hline
\end{tabular}

TABle IV. Metric Values of The EXAmple: Ambiguity Between ONE AND TWO COMMUNITIES.

\begin{tabular}{l||c|c|c|c}
\hline \hline & Modularity $(Q)$ & Split Penalty $(S P)$ & $Q_{s}$ & $Q_{d s}$ \\
\hline Two communities & 0.25 & 0.25 & 0 & 0.188 \\
\hline One community & 0 & 0 & 0 & 0.245 \\
\hline \hline
\end{tabular}

TABLE V. METRIC VALUes OF THE EXAMPLE: ONE WELL CONNECTED COMMUNITY.

\begin{tabular}{l||c|c|c|c}
\hline \hline & Modularity $(Q)$ & Split Penalty $(S P)$ & $Q_{s}$ & $Q_{d s}$ \\
\hline Two communities & 0.167 & 0.333 & -0.167 & 0.0417 \\
\hline One community & 0 & 0 & 0 & 0.23 \\
\hline \hline
\end{tabular}

TABLE VI. METRIC VALUES OF THE EXAMPLE: ONE VERY WELL CONNECTED COMMUNITY.

\begin{tabular}{l||c|c|c|c}
\hline \hline & Modularity $(Q)$ & Split Penalty $(S P)$ & $Q_{s}$ & $Q_{d s}$ \\
\hline Two communities & 0.0455 & 0.455 & -0.409 & -0.239 \\
\hline One community & 0 & 0 & 0 & 0.168 \\
\hline \hline
\end{tabular}

TABLE VII. Metric VAlues OF THE EXAMPLE: ONE Complete GRAPH.

\begin{tabular}{l||c|c|c|c}
\hline \hline & Modularity $(Q)$ & Split Penalty $(S P)$ & $Q_{s}$ & $Q_{d s}$ \\
\hline Two communities & -0.0714 & 0.571 & -0.643 & -0.643 \\
\hline One community & 0 & 0 & 0 & 0 \\
\hline \hline
\end{tabular}

in Figure 1. The seventh example is a complete graph with eight nodes and one big community containing all eight nodes while the alternative partition consists of the two small communities each containing four different nodes. We could easily judge that for the first, second, and the third examples, the community structure with two small communities is better than the community structure in which they are merged together. For the fourth example, the two different community structures are nearly of the same quality. However, for the fifth, sixth, and the seventh examples, the community structure with one big community is of better quality than the alternative.

Tables I-VII show the metric values of the seven network examples described above. Tables I-III, and Table VII demonstrate that modularity succeeds in measuring the quality of the two different community structures in those four examples. However, from Tables IV-VI, we could observe that modularity actually fails to measure the community quality of those three examples because it implies that the community structure with two small communities is better. In contrast, for the fifth and the sixth examples, the community structure with one big community is of better quality. Yet, in this case modularity gives preference to the community structure with two separated small communities, demonstrating that modularity has the problem of favoring small communities.

To address the drawback of favoring small communities, we propose that the quality of the community structure should take into account the edges between different communities. We introduce Modularity with Split Penalty $\left(Q_{s}\right)$ by subtracting from modularity the Split Penalty $(S P)$ which is the fraction of edges that connect nodes of different communities. More formally,

$$
Q_{s}=Q-S P .
$$

The intuition here is clear. Modularity measures the positive effect of grouping nodes together in terms of taking into account existing edges between nodes while Split Penalty measures the negative effect of ignoring edges joining members of different communities. Enlarging community eliminates some Split Penalty but if there are only a few edges across current partition, modularity of the merged community could be lower, negating the benefit of merging. Splitting a community into two or more communities introduces some Split Penalty but if there are only a few edges between those separated communities, an increase of modularity can make such splitting beneficial. Tables I-VII demonstrate that $Q_{s}$ can correctly measure the quality of the community structures of all seven network examples.

\section{Modularity with Split Penalty}

In this subsection, we extend the formula of $Q_{s}$ to different kinds of networks, such as unweighted and undirected networks, weighted networks, and directed networks, based on the corresponding formulas of modularity presented in Subsection III-III-A.

From Subsection III-III-B, we know that Split Penalty $(S P)$ is the fraction of edges that connect nodes of different communities. Thus, for undirected networks, no matter unweighted or weighted, Split Penalty is defined as

$$
S P=\sum_{c_{i} \in C}\left[\sum_{\substack{c_{j} \in C \\ c_{j} \neq c_{i}}} \frac{\left|E_{c_{i}, c_{j}}\right|}{2|E|}\right] .
$$

where $\left|E_{c_{i}, c_{j}}\right|$ is the number of edges from community $c_{i}$ to community $c_{j}$ for unweighted networks or the sum of the weights of the edges from community $c_{i}$ to community $c_{j}$ for weighted networks. For directed networks, Split Penalty is given by

$$
S P=\sum_{c_{i} \in C}\left[\sum_{\substack{c_{j} \in C \\ c_{j} \neq c_{i}}} \frac{\left|E_{c_{i}, c_{j}}\right|}{|E|}\right] .
$$

Therefore, for undirected networks, both unweighted and weighted, from Equations (1), (3), and (4), $Q_{s}$ is defined as

$$
Q_{s}=\sum_{c_{i} \in C}\left[\frac{\left|E_{c_{i}}^{\text {in }}\right|}{|E|}-\left(\frac{2\left|E_{c_{i}}^{\text {in }}\right|+\left|E_{c_{i}}^{\text {out }}\right|}{2|E|}\right)^{2}-\sum_{\substack{c_{j} \in C \\ c_{j} \neq c_{i}}} \frac{\left|E_{c_{i}, c_{j}}\right|}{2|E|}\right] \text {. }
$$

For directed networks, using Equations (2), (3), and (5), $Q_{s}$ can be expressed as

$$
\begin{aligned}
Q_{s}=\sum_{c_{i} \in C}[ & {\left[\frac{\left|E_{c_{i}}^{i n}\right|}{|E|}-\frac{\left(\left|E_{c_{i}}^{i n}\right|+\left|E_{\text {out }, c_{i}}\right|\right)\left(\left|E_{c_{i}}^{\text {in }}\right|+\left|E_{c_{i}, \text { out }}\right|\right)}{|E|^{2}}\right.} \\
& \left.-\sum_{\substack{c_{j} \in C \\
c_{j} \neq c_{i}}} \frac{\left|E_{c_{i}, c_{j}}\right|}{|E|}\right] .
\end{aligned}
$$




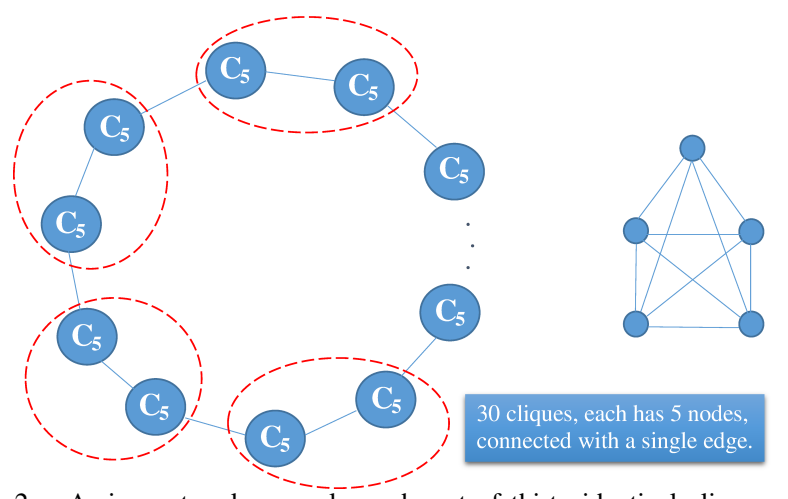

Fig. 2. A ring network example made out of thirty identical cliques, each having five nodes and connected by single edges.

\section{Motivation for Introducing Community Density}

Modularity has the resolution limit problem that $Q_{s}$ makes even worse. This problem is illustrated in Figure 2. It displays a ring network comprised of thirty identical cliques, each of which has five nodes and they are connected by single edges. In this case, the modularity of the community structure with each clique forming a different community, totally thirty communities, should be larger than that of the community structure in which two consecutive cliques form a different community, totally fifteen communities. However, Table VIII shows that the relation is reversed since the community structure with fifteen communities has larger modularity than that of the community structure with thirty communities. Moreover, as pointed out in [7], when $m(m-1)+2<n$, where $n$ is the number of cliques and $m$ is the number of nodes in each clique, modularity is higher for the large community with two consecutive cliques instead of the small community with a single clique. Moreover, Table VIII demonstrates that the difference of $Q_{s}$ for these two community structures is larger than the corresponding difference of modularity. More specifically, $\Delta Q_{s}=(0.8424-0.7848)=0.0576>\Delta Q=$ $(0.8879-0.8758)=0.0121$, which means that $Q_{s}$ makes the resolution limit problem even worse.

To address the resolution limit problem above, we propose and it is also quite intuitive to introduce community density into modularity, incorporating both the number of edges and the number of nodes in the communities and also Split Penalty. The corresponding new metric is called Modularity Density $\left(Q_{d s}\right)$. Table VIII shows that the $Q_{d s}$ of the community structure in which two consecutive cliques form a different community is almost half of the $Q_{d s}$ of the alternative in which each clique forms a different community. Hence, in this case, $Q_{d s}$ avoids the resolution limit problem. Furthermore, Tables I-VII and Figure 1 demonstrate that $Q_{d s}$ correctly measures the quality of the community structures of all seven network examples. Even for the network example of Figure 1(d) in which there is ambiguity which community structure is of higher quality, the $Q_{d s}$ of the one big community is only slightly larger than the $Q_{d s}$ of the two small communities as shown in Table IV.

\section{E. Modularity Density}

In this subsection, we will give the formulas for $Q_{d s}$ for different kinds of networks, including unweighted and undirected networks, weighted networks, and directed networks, based on the corresponding formulas of $Q_{s}$ presented in Subsection III-III-C.
TABLE VIII. METRIC VALUES OF THE EXAMPLE: A RING OF THIRTY CLIQUES, EACH HAVING FIVE NODES AND CONNECTED BY SINGLE EDGES.

\begin{tabular}{l||c|c|c|c}
\hline \hline & Modularity $(Q)$ & Split Penalty $(S P)$ & $Q_{s}$ & $Q_{d s}$ \\
\hline Thirty communities & 0.8758 & 0.09091 & 0.7848 & 0.8721 \\
\hline Fifteen communities & 0.8879 & 0.04545 & 0.8424 & 0.4305 \\
\hline
\end{tabular}

For undirected networks, regardless whether unweighted or weighted, we define $Q_{d s}$ using Equation (6) as follows

$$
\begin{aligned}
Q_{d s}= & \sum_{c_{i} \in C}\left[\frac{\left|E_{c_{i}}^{i n}\right|}{|E|} d_{c_{i}}-\left(\frac{2\left|E_{c_{i}}^{\text {in }}\right|+\left|E_{c_{i}}^{\text {out }}\right|}{2|E|} d_{c_{i}}\right)^{2}\right. \\
& \left.-\sum_{\substack{c_{j} \in C \\
c_{j} \neq c_{i}}} \frac{\left|E_{c_{i}, c_{j}}\right|}{2|E|} d_{c_{i}, c_{j}}\right] \\
d_{c_{i}}= & \frac{2\left|E_{c_{i}}^{i n}\right|}{\left|c_{i}\right|\left(\left|c_{i}\right|-1\right)} \\
d_{c_{i}, c_{j}}= & \frac{\left|E_{c_{i}, c_{j}}\right|}{\left|c_{i}\right|\left|c_{j}\right|}
\end{aligned}
$$

In the above, $d_{c_{i}}$ is the internal density of community $c_{i}, d_{c_{i}, c_{j}}$ is the pair-wise density between community $c_{i}$ and community $c_{j}$. Note that $\left|E_{c_{i}}^{i n}\right|$ in $d_{c_{i}}$ and $\left|E_{c_{i}, c_{j}}\right|$ in $d_{c_{i}, c_{j}}$ are unweighted for both unweighted and weighted networks, so that those two community densities are always less than or equal to 1.0.

For directed networks, using Equation (7), $Q_{d s}$ is given by

$$
\begin{aligned}
Q_{d s} & =\sum_{c_{i} \in C}\left[\frac{\left|E_{c_{i}}^{i n}\right|}{|E|} d_{c_{i}}-\sum_{\substack{c_{j} \in C \\
c_{j} \neq c_{i}}} \frac{\left|E_{c_{i}, c_{j}}\right|}{|E|} d_{c_{i}, c_{j}}\right. \\
& \left.-\frac{\left(\left|E_{c_{i}}^{i n}\right|+\left|E_{\text {out }, c_{i}}\right|\right)\left(\left|E_{c_{i}}^{i n}\right|+\left|E_{c_{i}, \text { out }}\right|\right)}{|E|^{2}} d_{c_{i}}^{2}\right], \\
d_{c_{i}} & =\frac{\left|E_{c_{i}}^{i n}\right|}{\left|c_{i}\right|\left(\left|c_{i}\right|-1\right)}, \\
d_{c_{i}, c_{j}} & =\frac{\left|E_{c_{i}, c_{j}}\right|}{\left|c_{i}\right|\left|c_{j}\right|} .
\end{aligned}
$$

\section{EVALUATION AND ANALYSIS}

In this section, we first introduce two real dynamic datasets and various other popular community quality measurements. Then, we show the experimental results that validate Modularity Density $\left(Q_{d s}\right)$ ability to address the two problems of modularity $(Q)$ simultaneously. Formal proofs that $Q_{d s}$ solves the modularity shortcomings are presented in the extended version of this paper [16].

\section{A. Real Dynamic Datasets}

In this subsection, we introduce two real dynamic datasets on which we conduct experiments in order to validate that $Q_{d s}$ avoids the two problems of modularity.

Senate Dataset [17], [18]. The Senate dataset is a timeevolving weighted network comprised of United States senators where the weight of an edge represents the similarity of their roll call voting behavior. This dataset was obtained from website voteview.com and the similarities between a pair of senators were calculated following Waugh et al. [18] as the number of bills for which the senators of the pair voted the same way, normalized by the number of bills for which they both voted. The dataset totally consists of 111 
TABLE IX. THE AVERAGE METRIC DIFFERENCES BETWEEN LABELRANKT WITH DIFFERENT VALUES OF CONDITIONAL UPDATE PARAMETER $q$ AND ESTRANGEMENT ON SENATE DATASET.

\begin{tabular}{|c|c|c|c|c|c|c|c|c|c|c|c|}
\hline LabelRankT conditional update $q$ & 0.05 & 0.1 & 0.2 & 0.3 & 0.4 & 0.5 & 0.6 & 0.7 & 0.8 & 0.9 & 0.95 \\
\hline$Q$ & -0.0534 & -0.0462 & -0.0408 & -0.0538 & -0.0714 & -0.0848 & -0.083 & -0.0897 & -0.0897 & -0.0848 & -0.08 \\
\hline$Q_{s}$ & -0.166 & -0.0802 & 0.0468 & 0.0808 & 0.0969 & 0.112 & 0.116 & 0.115 & 0.115 & 0.111 & 0.106 \\
\hline$Q_{d s}$ & -0.1638 & -0.0787 & 0.04847 & 0.08297 & 0.0995 & 0.1145 & 0.1182 & 0.1183 & 0.1183 & 0.1135 & 0.1083 \\
\hline \# Intra-edges & -159.102 & -32.444 & 234.296 & 387.38 & 510.645 & 616.855 & 615.123 & 624.764 & 624.764 & 602.627 & 580.733 \\
\hline Contraction & -6.806 & -3.023 & 2.481 & 4.553 & 5.937 & 7.033 & 7.065 & 7.227 & 7.227 & 6.927 & 6.622 \\
\hline \# Inter-edges & -75.962 & -54.098 & -123.898 & -187.99 & -245.198 & -299.356 & -300.108 & -303.043 & -303.043 & -292.782 & -282.442 \\
\hline Expansion & 6.448 & 2.91 & -2.428 & -4.416 & -5.737 & -6.847 & -6.878 & -7.009 & -7.009 & -6.724 & -6.431 \\
\hline Conductance & 0.213 & 0.0851 & -0.0886 & -0.148 & -0.186 & -0.214 & -0.216 & -0.224 & -0.224 & -0.213 & -0.201 \\
\hline
\end{tabular}

TABLE X. THE AVERAGE METRIC DIFFERENCES BETWEEN LABELRANKT WITH DIFFERENT VALUES OF CONDITIONAL UPDATE PARAMETER $q$ AND ESTRANGEMENT ON REALITY MINING BLUETOOTH SCAN DATA.

\begin{tabular}{|c|c|c|c|c|c|c|c|c|c|c|c|}
\hline LabelRankT conditional update $q$ & 0.05 & 0.1 & 0.2 & 0.3 & 0.4 & 0.5 & 0.6 & 0.7 & 0.8 & 0.9 & 0.95 \\
\hline$Q$ & -0.161 & -0.121 & -0.0783 & -0.0744 & -0.0724 & -0.0699 & -0.0702 & -0.0724 & -0.0742 & -0.0755 & -0.0774 \\
\hline$Q_{s}$ & -0.379 & -0.244 & -0.107 & -0.0802 & -0.0538 & -0.0497 & -0.0382 & -0.0405 & -0.0521 & -0.0634 & -0.0713 \\
\hline$Q_{d s}$ & -0.191 & -0.0984 & -0.0222 & -0.017 & -0.0116 & -0.0116 & -0.00318 & -0.00826 & -0.011 & -0.0115 & -0.0134 \\
\hline \# Intra-edges & -1450.893 & -956.006 & -479.377 & -331.371 & -230.263 & -183.536 & -102.94 & -78.93 & -155.183 & -242.287 & -333.419 \\
\hline Contraction & -86.909 & -69.914 & -52.543 & -46.371 & -43.176 & -40.567 & -35.948 & -36.425 & -38.006 & -41.277 & -45.425 \\
\hline \# Inter-edges & -39.949 & -76.524 & -159.74 & -167.333 & -190.947 & -190.865 & -196.098 & -193.123 & -188.708 & -179.653 & -178.96 \\
\hline Expansion & 52.529 & 25.829 & 6.289 & 5.76 & 5.664 & 7.07 & 4.881 & 6.799 & 6.916 & 6.117 & 5.669 \\
\hline Conductance & 0.23 & 0.176 & 0.114 & 0.1 & 0.0934 & 0.0933 & 0.0843 & 0.0955 & 0.102 & 0.107 & 0.104 \\
\hline
\end{tabular}

snapshots corresponding to Senate's activities over 220 years and includes 1916 unique senators.

Reality Mining Bluetooth Scan Data [19]. This dataset was created from the records of Bluetooth Scans generated among the 94 subjects in Reality Mining study conducted from 2004-2005 at the MIT Media Laboratory. In the network, nodes represent the subjects and the directed edges correspond to the Bluetooth Scan records while the weight of each edge represents the number of direct Bluetooth scans between the two subjects. In the experiments, we only used the records from August 02, 2004 (Monday) to May 29, 2005 (Sunday) and we divided them into weekly snapshots, so each snapshot represents scans collected during the corresponding week. There are total of 43 snapshots.

\section{B. Community Quality Measurements}

In the discussion of the experimental results we use various community quality metrics, including the number of Intraedges, Contraction, the number of Inter-edges, Expansion, and Conductance [8], which characterize how community-like is the connectivity structure of a given set of nodes. All of them rely on the intuition that communities are sets of nodes with many edges inside them and few edges outside of them. Now, given a network $G=(V, E)$ and given a community or a set of nodes $c$, let $|c|$ be the number of nodes in the community $c$ and let $\left|E_{c}^{i n}\right|$ denote the total number of edges in $c$ for unweighted networks or the total weight of such edges for weighted networks. We denote the total number of edges from the nodes in community $c$ to the nodes outside $c$ for unweighted networks or the total weight of such edges for weighted networks as $\left|E_{c}^{\text {out }}\right|$. Then, the definitions of the five quality metrics are as follows:

The number of Intra-edges: $\left|E_{c}^{i n}\right|$; it is the total number of edges in $c$ or the total weight of such edges. The larger the value of this metric is, the better the community quality is.

Contraction: $2\left|E_{c}^{i n}\right| /|c|$ for undirected networks or $\left|E_{c}^{i n}\right| /|c|$ for directed networks; it measures the average number of edges per node inside the community $c$ or the average weight per node of such edges. The larger the value of Contraction is, the better the community quality is.

The number of Inter-edges: $\left|E_{c}^{o u t}\right|$; it is the total number of edges from the nodes in community $c$ to the nodes outside $c$ or the total weight of such edges. The smaller it is, the better the community quality is.

Expansion: $\left|E_{c}^{\text {out }}\right| /|c|$; it measures the average number of edges (per node) that point outside the community $c$ or the average weight per node of such edges. The smaller the value of Expansion, the better the community quality is.

Conductance: $\frac{\left|E_{c}^{\text {out }}\right|}{2\left|E_{c}^{\text {in }}\right|+\left|E_{c}^{\text {out }}\right|}$ for undirected networks or $\frac{\left|E_{c}^{\text {out }}\right|}{E_{c}^{\text {in }}|+| E_{c}^{\text {out }} \mid}$ for directed networks; it measures the fraction of the total number of edges that point outside the community for unweighted networks or the fraction of the total weight of such edges for weighted networks. The smaller the value of Conductance is, the better the community quality is.

\section{Experimental Results}

In this subsection, we report the results of performing community detection on the two real dynamic datasets introduced in Subsection IV-A by using the dynamic community detection algorithms, LabelRankT [20] and Estrangement [17]. LabelRankT [20] detects communities in large-scale dynamic networks through stabilized label propagation. Estrangement [17] detects temporal communities by maximizing modularity in a snapshot subject to a constraint on the estrangement from the partition in the previous snapshot. We chose these two algorithms because the second algorithm relies on the modularity optimization while the first one does not. In the experiments, we adopted the best parameter of Estrangement but varying the conditional update parameter $q \in[0,1]$ of LabelRankT from 0.05 to 0.95 . As seen in the results, in most cases, the best $q$ is around 0.7 in agreement with the best value reported in [20]. For the community structures found by the two algorithms, we calculated the values of modularity $(Q)$, $Q_{s}$, Modularity Density $\left(Q_{d s}\right)$, and the five metrics described in Subsection IV-B.

Table IX and Table $\mathrm{X}$ present the average metric differences between LabelRankT with different values of conditional update parameter $q$ and Estrangement on Senate dataset and Reality Mining Bluetooth Scan data, respectively. That is, we first computed the values of the eight metrics above for the community detection results, detected by Estrangement, of each snapshot. Then, we calculated the eight metrics values for the community detection results, discovered by LabelRankT for all $q$, of each snapshot. Next, we got the metric differences of all eight metrics by subtracting the metric values of 
Estrangement from those of LabelRankT for all q's over each snapshot. Then, averaging those differences of each metric over all the snapshots, we obtained the corresponding average metric differences.

Table IX demonstrates that $Q$ gets its largest value when $q=0.2 ; Q_{s}$ reaches the largest value when $q=0.6 ; Q_{d s}$, Intra-edges, and Contraction get their largest values at $q=0.7$ and $q=0.8$; also, Inter-edges, Expansion, and Conductance reach their smallest values at $q=0.7$ and $q=0.8$. Thus, $Q_{d s}$ is consistent with the five metrics introduced in Subsection IV-B on determining the best $q$ for LabelRankT on Senate dataset while $Q$ and $Q_{s}$ are not consistent with them. Further, we could observe that $Q$ is always negative which indicates that LabelRankT performs below Estrangement over all $q$ 's because the goal of Estrangement is to maximize modularity $(Q)$. However, the other seven metrics imply that LabelRankT performs better than Estrangement when $q>0.1$. Therefore, we could explicitly observe that maximizing $Q$ to detect communities has problems in measuring the community detection quality correctly on Senate dataset.

Table $\mathrm{X}$ shows that six metrics get their best (largest or smallest) values at $q=0.6$ while the two exceptions, $Q$ and the number of Intra-edges, reach their largest values when $q=0.5$ and $q=0.7$, respectively. Thus, the six metrics, except $Q$ and the number of Intra-edges, are consistent on determining the best value of $q$ for LabelRankT on Reality Mining Bluetooth Scan data. This indicates that on Reality Mining Bluetooth Scan data, maximizing $Q$ to detect communities has problems.

It is also interesting to observe that for $q=0.05$ and $q=0.1$ in Table IX, Inter-edges metric implies that LabelRankT performs better than Estrangement on Senate dataset, which is not consistent with $Q_{s}, Q_{d s}$, Intra-edges, Contraction, Expansion, and Conductance metrics. Moreover, we could learn from Table X that all metrics, except Inter-edges metric, show that LabelRankT performs below the performance of Estrangement over all $q$ 's. Thus, Inter-edges metric has some problems. Also, as mentioned in the paragraph above, Intraedges metric is not consistent with the other six metrics on determining the best $q$ for LabelRankT, which means that Intra-edges metric has problems. We conjecture that the reason for the shortcoming of Intra-edges and Inter-edges metrics is the same as the case of $Q$ which does not consider the number of nodes in the communities. This reason also implies the superiority of $Q_{d s}$ over $Q$ and $Q_{s}$.

Based on the results presented in the above two tables, we conclude that $Q_{d s}$ solves the two problems of modularity. We also conjecture that the difference between the best values of $q$ for LabelRankT determined by $Q$ and $Q_{s}$ and the difference determined by $Q_{s}$ and $Q_{d s}$ on Senate dataset is a manifestation of the two problems of modularity maximization, namely favoring small communities and the resolution limit problem. Moreover, the difference between the best values of $q$ for LabelRankT determined by $Q$ and $Q_{s}$ on Reality Mining Bluetooth Scan data indicates that maximizing $Q$ has the problem of favoring small communities. Thus, $Q_{s}$ and $Q_{d s}$ can be used for checking whether finding communities by maximizing $Q$ on a specific dataset will suffer any of the two problems.

\section{CONCLUSION}

In this paper, we propose a new community quality metric, called Modularity Density, which solves the shortcomings of modularity of favoring small communities in some circumstances and large communities in others. We demonstrate with experiments on real dynamic datasets that Modularity Density is an effective alternative to modularity.

\section{ACKNOWLEDGMENT}

This work was supported in part by the Army Research Laboratory under Cooperative Agreement Number W911NF09-2-0053 and by the the Office of Naval Research Grant No. N00014-09-1-0607. The views and conclusions contained in this document are those of the authors and should not be interpreted as representing the official policies either expressed or implied of the Army Research Laboratory or the U.S. Government.

\section{REFERENCES}

[1] R. E. Park, Human communities: The city and human ecology. New York, NY: Free Press, 1952.

[2] W. S. Bainbridge, "Computational sociology," in Blackwell Encyclopedia of Sociology, 2007.

[3] M. E. J. Newman and M. Girvan, "Finding and evaluating community structure in networks," Phys. Rev. E, vol. 69, p. 026113, Feb 2004.

[4] S. Fortunato, "Community detection in graphs," Physics Reports, vol. 486, pp. 75-174, 2010.

[5] M. E. J. Newman, "Fast algorithm for detecting community structure in networks," Phys. Rev. E, vol. 69, p. 066133, Jun 2004.

[6] M. E. J. Newman, "Modularity and community structure in networks," Proceedings of the National Academy of Sciences, vol. 103, no. 23, pp. 8577-8582, 2006.

[7] S. Fortunato and M. Barthlemy, "Resolution limit in community detection," Proceedings of the National Academy of Sciences, vol. 104, no. 1, pp. 36-41, 2007.

[8] J. Yang and J. Leskovec, "Defning and evaluating network communities based on ground-truth," IEEE International Conference On Data Mining (ICDM), 2012

[9] M. E. J. Newman, "Analysis of weighted networks," Phys. Rev. E, vol. 70, p. 056131, Nov 2004.

[10] E. A. Leicht and M. E. J. Newman, "Community structure in directed networks," Phys. Rev. Lett., vol. 100, p. 118703, Mar 2008.

[11] J. W. Berry, B. Hendrickson, R. A. LaViolette, and C. A. Phillips, "Tolerating the community detection resolution limit with edge weighting," Phys. Rev. E, vol. 83, p. 056119, May 2011.

[12] B. H. Good, Y.-A. de Montjoye, and A. Clauset, "Performance of modularity maximization in practical contexts," Phys. Rev. E, vol. 81, p. 046106, Apr 2010.

[13] J. Reichardt and S. Bornholdt, "Statistical mechanics of community detection," Phys. Rev. E, vol. 74, p. 016110, Jul 2006.

[14] A. Arenas, A. Fernndez, and S. Gmez, "Analysis of the structure of complex networks at different resolution levels," New Journal of Physics, vol. 10, no. 5, p. 053039, 2008.

[15] A. Lancichinetti and S. Fortunato, "Limits of modularity maximization in community detection," Phys. Rev. E, vol. 84, p. 066122, Dec 2011.

[16] M. Chen, T. Nguyen, and B. K. Szymanski, "A new metric for quality of network community structure," ASE Human Journal, to appear, 2013.

[17] V. Kawadia and S. Sreenivasan, "Sequential detection of temporal communities by estrangement confinement," Scientific Reports, vol. 2, November 2012

[18] A. S. Waugh, L. Pei, J. H. Fowler, P. J. Mucha, and M. A. Porter, "Party polarization in congress: A network science approach," $\operatorname{arX}$ iv:0907.3509, 2010.

[19] N. Eagle, A. Pentland, and D. Lazer, "Inferring social network structure using mobile phone data," Proceedings of the National Academy of Sciences (PNAS), vol. 106, no. 36, pp. 15 274-15278, 2009.

[20] J. Xie, M. Chen, and B. K. Szymanski, "LabelrankT: Incremental community detection in dynamic networks via label propagation," in ACM SIGMOD Workshop on Dynamic Networks Management and Mining (DyNetMM), New York, USA, 2013. 